\title{
Consumo tecnológico: Análisis de los determinantes del equipamiento doméstico mediante Arboles de Decisión
}

\author{
Technological consumption: Analysis of the determinants of household equipment \\ through Decision Trees
}

\section{Carlos Rodriguez Garcés ${ }^{1}$ y Daniela Sandoval Muñoz ${ }^{2}$}

Resumen: El proceso de tecnificación del hogar como consecuencia del masivo acceso al consumo posibilitado por el desarrollo tecnológico y el mejoramiento de las rentas, brinda una mejor calidad de vida, confort y status, además de transformar los usos del tiempo, hábitos y roles al interior del espacio doméstico. Haciendo uso de la base de datos del Centro de Estudios Públicos para los años 2004-2013 en Chile, se realiza un análisis de tendencia y de segmentación mediante árboles de clasificación de los niveles de penetración tecnológica en el espacio doméstico y los factores discriminantes. Los resultados dan cuenta de una rápida y masiva incorporación de dispositivos tecnológicos, no obstante constatarse ciertos rezagos en razón del tipo de dispositivo y perfil del usuario, siendo el vector de mayor segmentación el nivel socioeconómico. Se observan aparatos de acceso universal y perfectamente integrados, tales como el teléfono celular; otros de acelerada irrupción que amenazan hegemonías tecnológicas de antaño, tal es el caso de la televisión por cable o satelital.

Palabras clave: Tecnificación, elitización, equipamiento doméstico, consumo tecnológico, electrodoméstico.

Abstract: The process of modernization of the home as a result of mass access to consumption
made possible by technological development and improving incomes, providing a better quality
of life, comfort and status in addition to transform the use of time, habits and roles within the
domestic space. Using the database of the Center for Public Studies for the years 2004-2013 in
Chile, trend analysis and segmentation by classification trees levels of technology penetration
in the domestic space and discriminating factors is performed. The results show a rapid and
massive incorporation of technological devices, however constatarse certain lags because of the
type of device and user profile, with greater segmentation table socioeconomic status. Universal
devices and seamlessly integrated, such as cell phone access have been satisfied; other
accelerated technological breakthrough that threaten hegemonies of old, as in the case of cable
or satellite.

Keywords: Modernization, gentrification, elitism, household equipment, technological consumer, appliance.

1 Doctor en Multimedia Educativa por la Universidad de Barcelona. Catedrático en la Universidad del Bío- Bío de Chile. Director del Centro de Investigación y Desarrollo en Informática Educativa (CIDCIE). Chile. E-mail: carlosro@ubiobio.cl

2 Investigadora del Centro de Investigación y Desarrollo en Informática Educativa (CIDCIE) de la Universidad del Bío-Bio de Chile. E-mail: dfsandov@alumnos.ubiobio.cl

Recibido: 30/12/2014; Aceptado: 28/05/2015. http://dx.doi.org/10.18004/riics.2015.julio.70-85

Rev. Int. Investig. Cienc. Soc.

Vol. $11 \mathrm{n}^{\circ} 1$, julio 2015. pág. 70-85.

ISSN (Impresa) 2225-5117. ISSN (En Linea) 2226-4000. 


\section{INTRODUCCIÓN}

Las familias son unidades de producción y consumo que en la búsqueda de satisfacer sus necesidades adquieren bienes y servicios que les proporcionen un mayor nivel de satisfacción. Las personas como integrantes de esta unidad tranzan ocio, venden tiempo en el mercado del trabajo y desarrollan variadas estrategias en la obtención del ingreso que posibilite determinados niveles de consumo.

Si bien estos atributos de individuos y grupos han acompañado a la historia de la humanidad en su conjunto, es precisamente en las sociedades actuales donde el consumo de masas se ha generalizado, diversificado e intensificado, adquiriendo patrones de comportamiento que se distancian de la mera satisfacción de necesidades básicas o búsqueda de la subsistencia. El ascenso masivo al consumo ha sido posibilitado por el desarrollo tecnológico, el aumento de las rentas y un cambio de prioridades que redefinen lo suntuario, donde los lujos del pasado son hoy considerados como objetos ordinarios (Bell, 2004).

En efecto, el desarrollo tecnológico sin precedentes iniciado en la década del veinte del siglo pasado en los Estados Unidos y en algunos países de Europa Occidental, posibilitó que una masiva producción de bienes se llevara a cabo de forma rápida y menos costosa. Adicionalmente, se produjo un crecimiento $\mathrm{y}$ redistribución de las rentas, hecho que contribuyó a que las personas contaran con un excedente monetario que, además de satisfacer sus necesidades básicas, les permitiera gastar en bienes de consumo diversos, muchos de ellos de perfil tecnológico.

A ello se suma un fuerte impulso de los medios de comunicación como una herramienta publicitaria (Brandle, 2008) persuadiendo a audiencias cada vez más masivas, modificando sus pautas de consumo e influyendo en sus opciones de compra. Tecnología, ingresos y publicidad que, con matices y rezagos, instalan la sociedad del consumo a nivel planetario. Acceso que no solo mejora la calidad de vida de las personas, sino que además llega a reemplazar los antiguos valores del trabajo y la austeridad (Brandle, 2008).

Para que la sociedad de consumo se instale se precisa de un sistema productivo que presente una variada oferta para satisfacer las demandas de los consumidores y una apropiada distribución de la renta que haga que la capacidad de compra se distribuya más homogéneamente entre los estratos de la sociedad (Díaz de Rada, 2001). Las sociedades modernas articulan producción y consumo de masas a través de la fuerza del trabajo que se emplea en las fábricas, la que mediante una retribución económica o salario genera capacidad adquisitiva o de endeudamiento a través del crédito. 
La sociedad de consumo es, en consecuencia, un fenómeno fuertemente asociado, aunque no en exclusividad, a procesos de urbanización. Las metrópolis no solo concentran una gran masa de población e industrias, sino que además proporcionan un amplio espacio para imaginar y crear nuevas formas de vida, relaciones sociales, experiencias e identidades inspiradas en el confort y bienestar que las mercancías prometen (Carosio, 2008).

El mundo rural por su parte, no permanece ajeno a las transformaciones que de este paradigma societal se derivan. La movilidad espacial temporal o permanente para acceder a trabajo o servicios como el educativo no tan solo involucra un reordenamiento territorial, sino que expone a la población rural a profundos cambios en la identidad del linaje, la cultura del trabajo, roles familiares, entre otros.

En ambos contextos estas transformaciones son comandadas por la hegemonía del mercado en la cultura de la urbanidad (Santa Marina, 2001), siendo precisamente las ciudades el escenario que posibilita el giro cultural hacia la sociedad de consumo (Carosio, 2008). Los dispositivos tecnológicos, como expresión de urbanidad, modifican las relaciones sociales incluso al interior del grupo familiar, donde pese a compartir el mismo espacio se evidencian tendencias a la privatización e individualización de los usos culturales (Boito, Michelazzo, y Conicet, 2011).

En consecuencia, es objetivo de este artículo explorar la irrupción tecnológica en un espacio temporal de diez años, constatando el comportamiento diferencial que tienen dichos dispositivos tecnológicos y los factores que condicionan su mayor o menor presencia al interior del hogar. Ello en el entendido que, la penetración tecnológica en el hogar es un representativo indicador que posibilita ilustrar el cambio societal que modifica pautas de comportamiento en la búsqueda de confort, bienestar y status.

Los dispositivos tecnológicos están cada vez más presentes en variedad y cantidad en el espacio doméstico, con niveles de penetración que, aún siendo diferenciados, reducen las brechas, redefinen lo exclusivo y las formas de vida elitizadas. Masiva incorporación de tecnologías ahorradoras de tiempo y consumidoras del mismo, que liberan espacio para el ocio posibilitando su inversión en otras actividades sociales o productivas.

En efecto, el acceso tecnológico aumenta la productividad del hogar y con ello hace menos necesaria la presencia permanente de la mujer en las tareas domésticas, incentivando su participación en el mercado del trabajo. Participación que por otra parte, se hace más imprescindible como mecanismo eficiente para allegar ingresos que mejoren las rentas familiares que permiten sostener el acceso a bienes y servicios, expresión de confort, bienestar y status. 


\section{METODOLOGIA}

Estudio de carácter cuantitativo que a través del análisis de base de datos preexistentes, tiene como objetivo cuantificar los niveles de penetración de dispositivos tecnológicos en el espacio doméstico en el transcurso de una década (2004-2013), así como los factores que en niveles de importancia diferenciados segmentan y condicionan dicha penetración. Como insumo se utilizó la Encuesta CEP de los años respectivos, instrumento diseñado y aplicado por el Centro de Estudios Públicos de Chile a una muestra representativa de habitantes del territorio chileno.

El universo de estudio está definido por la población de 18 años y más de zonas urbanas y rurales. El muestreo es probabilístico estratificado por conglomerados múltiples entrevistándose a una muestra de alrededor de 1.500 personas en cada año, con un error de muestreo del $\pm 3 \%$ y un nivel de confianza del $95 \%$, estableciéndose un margen de respuesta efectiva promedio para todos los años cercano al $85 \%$.

Las bases de datos fueron sometidas a un proceso de manipulación segmentando la unidad de análisis y recategorizando variables según requerimientos. Se construye con cada una de ellas un índice que da cuenta del nivel de penetración de los dispositivos tecnológicos y electrodomésticos existentes en el hogar denominado Índice de Tecnificación del Hogar (ITH). Indicador cuantitativo escalar que expresa la proporción de un conjunto de 15 aparatos electrónicos estándar disponibles y en uso en el hogar, el cual transita entre 0 , ausencia absoluta, y 1 , que da cuenta de la presencia total de los dispositivos en el espacio doméstico.

En primera instancia se realiza un análisis longitudinal de carácter descriptivo a fin de conocer el comportamiento de la penetración tecnológica en el espacio doméstico, junto con un análisis desagregado por tipo de dispositivo. En una segunda instancia se realiza un análisis multivariante a través de Árboles de Clasificación o de Decisión. Esta es una técnica exploratoria de datos (data mining) consistentes en el estudio y reducción de grandes volúmenes de información con el propósito de descubrir patrones predictivos o explicativos del proceso de tecnificación o penetración en el hogar.

Estos árboles, construidos mediante un algoritmo, van subdividiendo recursivamente en nodos los casos u observaciones de la base de datos, de forma tal que en cada subdivisión las frecuencias relativas de las categorias de la variable dependiente vayan tendiendo a 0 ó a 1 , utilizando para ello el método CHAID, un detector automático mediante Chi Cuadrado para determinar las divisiones óptimas. Se opta por este modelo de Árbol de Clasificación por su transparencia intuitiva y fácil interpretación, además de utilizar indistintamente variables categóricas y 
continuas. De hecho, es recomendable por sobre otras técnicas de clasificación dado que su uso con variables independientes politómicas posibilita la generación de mejores resultados. Esto permitió configurar el Índice de Tecnificación del Hogar (ITH) con base a las características o atributos distintivos y discriminantes.

\section{RESULTADOS Y DISCUSIÓN}

Una de las dimensiones configuradoras del bienestar doméstico se relaciona con el nivel de equipamiento del hogar, aspecto que convive con las condiciones de habitabilidad, dotación de servicios básicos, distribución y disponibilidad de los espacios; los cuales en su conjunto determinan el confort y calidad de vida de la familia al interior del espacio doméstico. La dotación tecnológica a su vez, es expresión de las nuevas pautas de consumo y estilos de vida presentes en la sociedad moderna que dan cuenta de un proceso de estetización de la vida cotidiana donde el objeto tecnológico trasciende su función meramente utilitaria o material.

Ambos componentes, búsqueda de confort y nuevas pautas de consumo, son causa y efecto del proceso de equipamiento del hogar. Evento que se busca ilustrar a través de la construcción de un índice de tecnificación del hogar (ITH), esto es, la proporción de un set de 15 aparatos electrónicos disponibles y en uso en el espacio doméstico. Índice que transita desde el 0 , ausencia absoluta, hasta el 1, que expresa la presencia de todos los dispositivos en la totalidad de los hogares.

$\mathrm{El}$ análisis tendencial o comportamiento de este índice da cuenta de un aumento significativo en el decenio analizado (Figura 1). En el año 2004 el índice ITH en los hogares chilenos alcanzaba un 0,48 , esto es, existian en promedio al menos uno de cada dos dispositivos en los hogares. Al año 2013, en cambio, para igual número de aparatos, se registraba un índice de 0,60 .

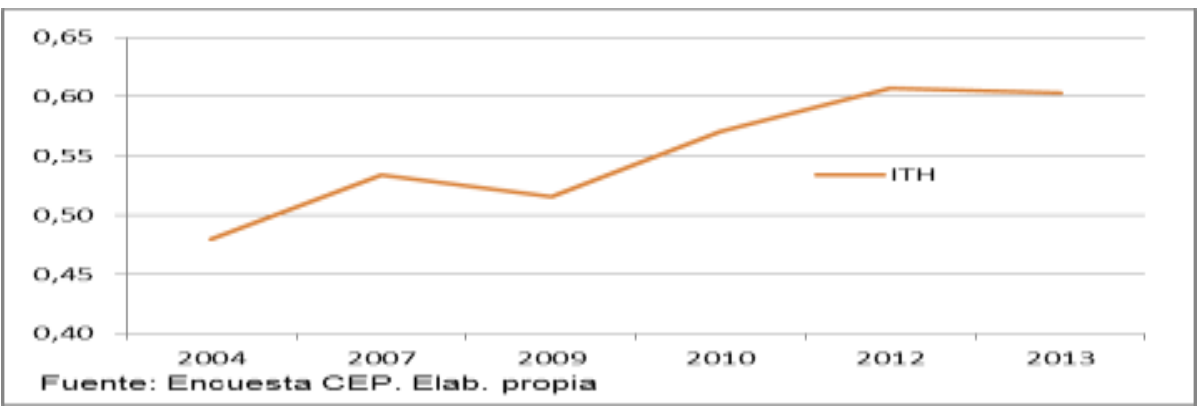

Figura 1. Índice de Tecnificación del Hogar 2004-2013. 
Ello significa que en estos escasos diez años los aparatos tecnológicos han aumentado su presencia en un $25 \%$ en promedio. Penetración tecnológica importante si se toma en consideración que éste es un índice resumen que no da cuenta de la dispersión por tipo de hogar ni menos la heterogénea distribución existente por tipo de aparato.

Este acelerado y masivo proceso de tecnificación del hogar tiene directas implicancias sociales en razón del mejoramiento de las condiciones de habitabilidad de la vivienda. Un mayor equipamiento se asocia a un mayor confort del espacio privado familiar haciendo que las personas pasen más tiempo en él, situación que modifica las pautas de sociabilidad, así como la estructura familiar misma. La tecnificación del hogar releva en importancia al espacio doméstico como lugar donde se pasa gran parte del tiempo permitiendo asociar y fortalecer aspectos simbólicos a la primigenia función de refugio atribuido a la vivienda. El hogar no solo es un reducto de intimidad y protección frente al otro amenazante, sino que además es un espacio-objeto estilizado donde la tecnificación es expresión de status, prestigio y diferenciación social.

Los aparatos tecnológicos además, tienen la capacidad de modificar las relaciones sociales y pautas de comportamiento de forma que la presencia de determinados dispositivos tecnológicos no tan solo retrotrae al individuo al espacio de lo doméstico, en perjuicio de lo público, sino que además disgrega a los miembros al interior del hogar. Aparatos como la radio o la televisión, como antes la luz eléctrica, pierden la capacidad de antaño de reunir a la familia. Estos dispositivos aumentan en número dentro del hogar e irrumpen nuevas aplicaciones, todo lo cual posibilita que los distintos miembros de la familia puedan realizar actividades diversas en diferentes habitaciones a pesar de compartir un mismo espacio (Livingstone, 1992).

Por otra parte, la familia como institución constituyente del espacio social y constantemente permeada e influenciada por los hechos ocurridos en dicho entorno, se ve relacionada bidireccionalmente con el proceso de tecnificación. El consumo de tecnologías en el espacio doméstico facilitan las tareas del hogar, generalmente asociados a la imagen femenina, aumentando los niveles de confort y bienestar. Mayor comodidad que al precisar de mayores ingresos, capaces de solventar adquisición y mantención de determinados bienes y servicios, conminan a aumentar el número de perceptores de ingresos del hogar dado que las nuevas pautas de consumo y estándar de vida, al menos en lo que a clase media se refiere, requiere de dos salarios (Senet, 2000). La familia, como unidad de consumo, haría uso de su fuerza de trabajo de reserva o capacidad ociosa, condición de inactividad principalmente asociada a las mujeres. En consecuencia, estas nuevas necesidades 
son las que impulsan también la irrupción de la mujer al mercado del trabajo (Brandle, 2010), al ser éste un contingente ahora no tan solo más preparado, sino que además liberado de las ataduras del rol doméstico de carácter reproductivo.

En efecto, gracias a la incorporación de elementos tecnológicos como la lavadora, la centrifuga o secadora, las funciones que cumplía la mujer al interior del hogar se ven enormemente simplificadas, en lo que concierne a facilidad para llevarlas a cabo y más importante aún, un significativo ahorro de tiempo. Ahorro de tiempo que impulsa a la mujer a salir del espacio privado y buscar oportunidades de trabajo remunerado en lo público; hecho que altera las pautas al interior del grupo familiar e inciden, no sin cierta resistencia, en una redefinición de roles.

Estas nuevas pautas de consumo y expresión de confort mediado por la adquisición tecnológica no tan solo pueden tensionar la convivencia familiar, sino que además, inciden en la configuración de la estructura familiar misma. En efecto, la noción de vivir bien, en un espacio confortable y urbano, siendo una actitud general frente a la sociedad, el trabajo y las relaciones sociales, evidencia sus manifestaciones particulares en el contexto familiar y doméstico. Las personas con el fin de garantizar dicho ideal acumulan capital humano, retardan la nupcialidad, postergan y planifican la llegada de los hijos, se reduce el tamaño de las familias. No obstante la importancia que revisten los procesos de tecnificación del hogar por su uso instrumental y simbólico, con dispositivos "ahorradores de tiempo" y de "consumo de tiempo", los índices de penetración de manera desagregada manifiestan un comportamiento temporal heterogéneo.

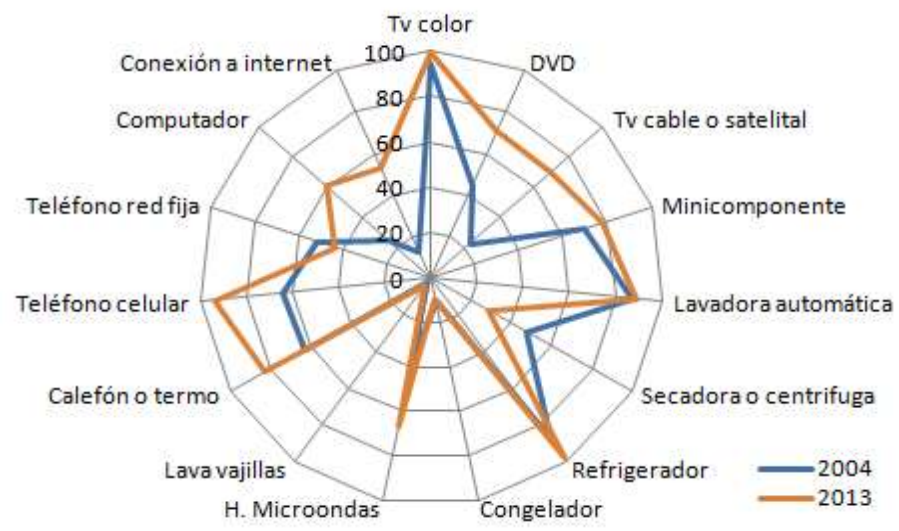

Figuar 2. Penetración Tecnológica del Hogar 2004-2013. Fuente: Encuesta CEP. Elaboración propia. 
Si bien la figura 2 ilustra un notorio incremento del equipamiento doméstico, puestos a analizar cómo se configura la presencia de los distintos dispositivos tecnológicos en los hogares chilenos se observa un comportamiento diferenciado de éstos en razón de su tipología. Al año 2013, con excepción de la secadora o centrífuga y teléfono de red fija, todos han aumentado sus niveles de penetración dentro del espacio domiciliario, constituyéndose en aparatos tecnológicos plenamente integrados como medios de consumo. El televisor a color, teléfono celular, refrigerador y la lavadora, evidencian una presencia masiva, en algunos casos con penetración casi universal con atenuadas distinciones por grupo social de pertenencia. Otros en cambio, tienen niveles de penetración mucho más acotados y segmentados. Dispositivos como el lavavajillas y el congelador registran una disponibilidad mucho más reducida y marginal.

Por otra parte, la tecnificación del espacio domiciliario, si bien no es un fenómeno reciente, es en la actualidad donde este proceso se acelera, masifica y diversifica. Los hogares disponen de una mayor variedad de dispositivos, algunos en más de una unidad por hogar, incluso aparatos que hace diez años eran inexistentes al interior del espacio domiciliario o considerados un lujo o bien suntuario hoy se constituyen en un objeto de uso común. La televisión por cable o satelital, el computador, la internet, el DVD y el teléfono celular son expresión de este fenómeno y dan cuenta del cada vez más atenuado lapso temporal que transita la tecnología desde la creación innovativa a la masificación como objeto de consumo. Penetración alentada por el abaratamiento de los costos de producción, la disponibilidad de ingresos generados principalmente por el trabajo, el acceso al crédito sustentado en la capacidad de endeudamiento avalada en la formalidad y regularidad de las rentas laborales, así como a las nuevas necesidades demandadas por los nuevos estilos de vida generados.

El éxito en la penetración dentro del espacio domiciliario que evidencian estos dispositivos los constituye en íconos de los procesos de tecnificación a pesar de su reciente invención, es explicado además por una serie de atributos, algunos de carácter subjetivo que los usuarios le reconocen y valoran de forma de inclinar su opción de compra hacia ellos. Son aparatos que emulando a los preexistentes, prometen ahora una funcionalidad distintiva que los distancia de sus antecesores, brindando esta vez al usuario un conjunto de posibilidades y opciones que antes le estaban negadas. Los computadores e internet por ejemplo, no tan solo permiten el acceso a la información y la comunicación sino que además es una poderosa herramienta para la entretención, reuniendo en sí misma toda una gama de funcionalidades y contenidos, espacio virtual perfectamente integrado en la 
configuración del tiempo libre, incluso para la educación y el trabajo que se constituye en una suerte de extensión de lo social.

La televisión por cable o satelital por su parte, inspirada en la televisión analógica por vía terrestre, supera a ésta última ampliamente, brindando ahora al usuario la posibilidad de elegir los contenidos y horarios liberándolo de las restricciones de antaño. Libertad, como atributo reconocido del dispositivo, que es fuertemente valorada por el usuario y potenciada por la convergencia tecnológica, esto es, la tendencia cada vez más masificada de que diferentes sistemas tecnológicos evolucionen hacia la realización de un conjunto de tareas diferentes alojadas en un mismo dispositivo. Resignificación funcional que acaba utilizando la tecnología para propósitos muy diferentes a aquellos que concibieron los diseñadores que le dieron origen (Castells, 2006).

La penetración de algunos dispositivos se hace también a expensas de otros en un contexto de sustitución tecnológica cada vez más generalizada en la medida que las nuevas tecnologías no solo son más económicas sino que además polivalentes y versátiles que trascienden su funcionalidad primigenia. Así por ejemplo, el teléfono fijo pierde el protagonismo de antaño con la aparición del teléfono móvil. En el año 2004 un 51,7\% de las personas señalaban disponer de un teléfono de red fija, cifra que se reduce a un 43,4\% en el año 2013. A su vez, el teléfono móvil aumenta su presencia de un $64 \%$ a un $93,7 \%$ en igual periodo, registrando una gran homogeneidad por zona geográfica, estrato socioeconómico y sexo.

Dispositivo de acceso universal que brinda la posibilidad de la conexión perpetúa junto con la promesa de un uso que trasciende la mera actividad comunicativa con base a un conjunto de aplicaciones integradas. Elementos que dan valor agregado a la telefonía móvil reforzando el abanico de posibilidades que poseen los usuarios para potenciar o complementar el servicio telefónico elemental (teléfonos móviles que funcionan como micro computadores, con cámaras digitales de alta resolución, reproductores de audio, etc.). Polifuncionalidad que potencia su valor como signo de marcado carácter individual o privatizado al posibilitar consumos individuales aún en espacios compartidos (Boito, Michelazzo, y Conicet, 2011), situación que conlleva aspectos culturales y simbólicos de relevancia en la vida cotidiana cuyos impactos recién buscamos dilucidar.

Por último, dentro del espacio domiciliario se distinguen un conjunto de dispositivos escasamente integrados, de uso marginal a nivel global y presencia fuertemente elitizada. La secadora, el lavavajilla y el congelador son íconos representativos de esta tendencia siendo expresión de estilos de vida elitizados que valoran fuertemente el confort y asignan importancia al tiempo como recurso escaso, 
donde la disponibilidad tecnológica viabiliza nuevos estilos de vida, permitiendo costumbres de uso con mayor facilidad y rapidez. Así por ejemplo el congelador permite el almacenamiento y preservación de grandes cantidades de alimentos, evitando la compra diaria o presencia estacional de éstos.

No obstante lo anterior, los desequilibrios en la relación costo beneficio asociados a dichos dispositivos estarian en la base explicativa de su escasa penetración domiciliaria. En efecto, son tecnologías costosas, circunscritas a una función única, abocados a la realización de una tarea cuya sustitución no ofrece las ventajas comparativas que la inversión demanda. Son tecnologías que se concentran casi en exclusividad en los hogares de mayores ingresos, preferentemente urbanos, cuyas viviendas disponen del espacio físico que su instalación requiere, aspecto este último que se constituye en un desincentivo a la inversión dada la tendencia al reducido espacio con los que cuentan las viviendas modernas.

A pesar de las diferencias individuales en los niveles de penetración por tipo de dispositivo tecnológico, se observa que los hogares, como unidad de consumo, orientan sus elecciones e inversión tecnológica hacia un conjunto de componentes que, en una relación de costo beneficio, posibilita por un lado el ahorro de tiempo, y por el otro el consumo del mismo.

Determinado equipamiento tecnológico en el hogar posibilita un ahorro significativo de tiempo aumentando la capacidad productiva familiar. Hecho especialmente notorio en las tecnologias creadas en los 70' y masificadas en Chile a partir de los años 80'. Ejemplo ilustrativo de ello es la lavadora, especialmente la automática, elemento de alta penetración. Estos dispositivos optimizadores de la producción doméstica en los hogares de mayores recursos, posibilita un trasvase de funciones tercerizadas o delegadas a una entidad o persona externa al grupo familiar para restituirla como función propia a éste, sin que ello implique una sobrecarga indeseada. Por otro lado, en los grupos familiares de nivel socioeconómico medio y bajo, implican una función de sustitución liberando a la persona del grupo familiar que por costumbre realizaba dicha tarea, responsabilidad que en casi exclusividad recaía sobre la mujer, en especial la dueña de casa.

El impacto que tiene la penetración de este tipo de aparatos ahorradores de tiempo en el hogar tributa directamente en la disponibilidad de tiempo de ocio el cual puede ser invertido en actividades extra domésticas tales como el trabajo remunerado, incluso la educación. Así mismo, su uso reduce la monotonía y el desgastador esfuerzo que implicaba muchas de las tareas del quehacer doméstico, constituyéndose su adquisición en un componente con ribetes de reivindicación emancipadora, no solo por liberar tiempo sino también por ahorrar trabajo 
simplificando las tareas del hogar (Robinson y Godbey, 2008). Es esta relación de ocio (tiempo libre) y trabajo (menor esfuerzo) como atributos asociados al equipamiento tecnológico lo que explica que, una vez superadas las restricciones presupuestarias o de ingresos, no se observen diferencias significativas en el nivel de equipamiento del hogar en razón de si la mujer participa del mercado laboral o por el contrario se dedica exclusivamente a las labores domésticas.

Este ahorro de tiempo tiene su correlato en el espacio extra doméstico o económico productivo. El desarrollo tecnológico aumentó la productividad necesitando de menor tiempo de trabajo por unidad producida lo que derivó en un incremento de ocio disponible, "espacio vacío" que se busca ocupar y donde las tecnologías proporcionan una alternativa eficiente que no precisa de la implicación directa de la persona en su gestión, sino que hace de ésta un consumidor pasivo (Corvalán, 2012).

Así constatamos un conjunto de tecnologías consumidoras de tiempo de ocio, atributo muy extendido pero de calidad diferenciada dependiendo de los niveles de preparación e ingreso (Brandle, 2008). La adquisición y uso de minicomponentes, la televisión, tanto la genérica, satelital o por cable, las consolas, el computador e internet, e incluso el mismo teléfono móvil son expresión de esta tendencia que irrumpe en la población en general pero especialmente en los sectores más jóvenes. Estas tecnologías desarrollan sus intereses y necesidades asociadas a la entretención y el ocio en un espacio de libertad e independencia.

En atención a que la gran mayoría de las tecnologías en su doble condición de ahorradoras de tiempo y consumo del mismo son de movilidad restringida limitando su existencia y uso en los contornos del espacio doméstico tanto como para ser considerado equipamiento del hogar en sus dimensiones línea blanca y línea gris, y siendo precisamente la mujer quien tiene o influencia la capacidad de compra hacia determinados productos es que se ha considerado relevante analizar cuáles son los factores que, desde el perfil femenino y en sus distintos niveles de importancia, influyen en el proceso de tecnificación del hogar.

El Árbol de clasificación (Figura 3) ilustra que en la configuración de la penetración tecnológica del hogar (ITH) el factor con mayor capacidad de segmentación es el nivel socioeconómico. En los sectores de menores recursos, que corresponde al nivel socioeconómico bajo, los índices de penetración son significativamente menores que en los grupos del los niveles socioeconómicos alto y medio ( $\mu=0,47 ; \sigma=0,18$ versus $\mu=0,7 ; \sigma=0,16$ respectivamente). Hecho que refuerza la hipótesis de la brecha tecnológica por la vía socioeconómica, constituyendo ésta uno 
de los factores de mayor peso explicativo al momento de dar cuenta del desarrollo desigual de la irrupción tecnológica al interior del hogar.

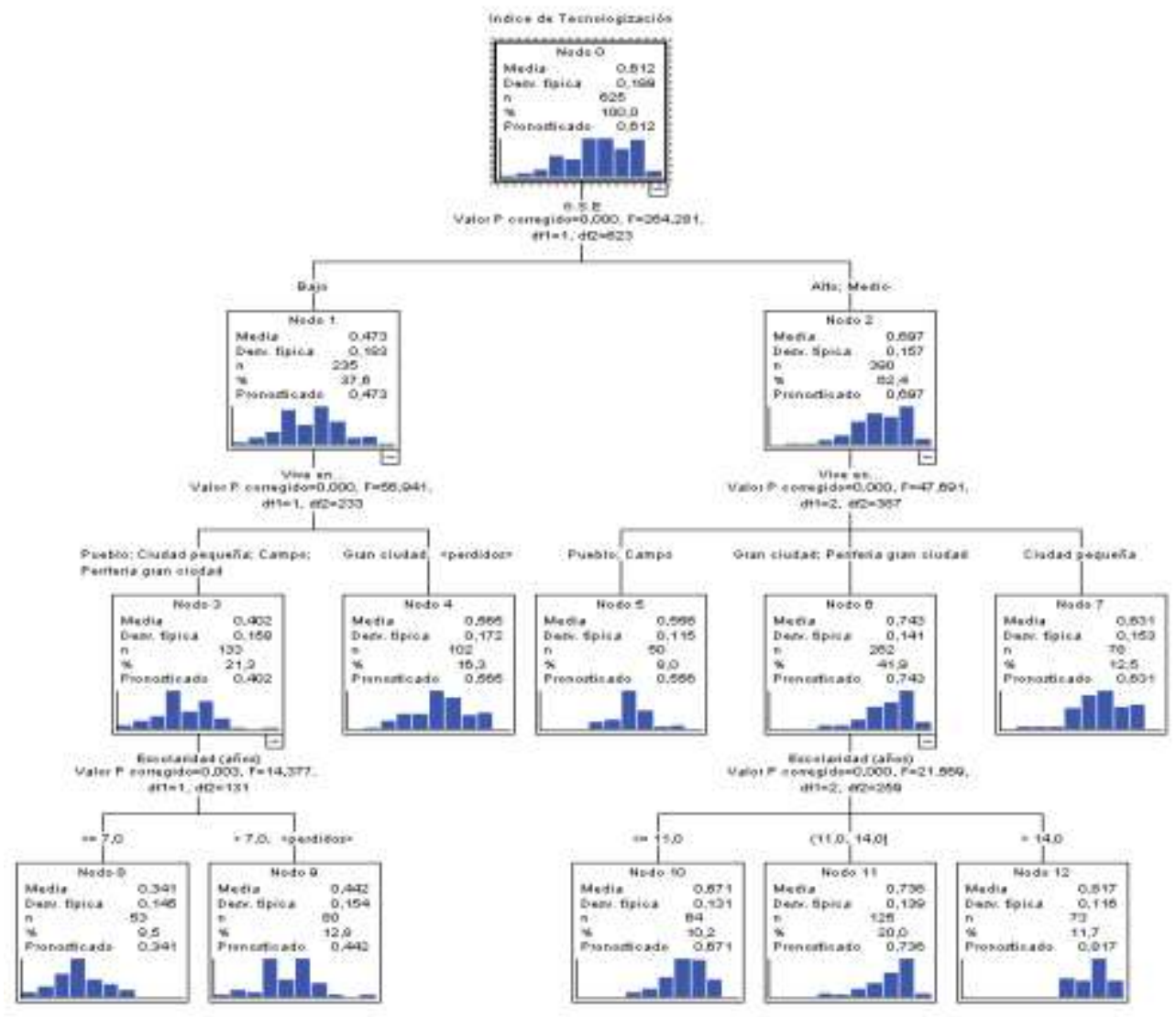

Figura 3. Árbol de Clasificación: Predictores de la Tecnificación del Hogar en la población femenina.Fuente: Encuesta CEP. Elaboración propia.

En un segundo nivel de segmentación y para ambos grupos aparece como relevante el factor Zona de Procedencia. Para el grupo de rezagados, esto es NSE bajo, se constatan como elemento diferenciador vivir en una ciudad pequeña, pueblo o campo respecto de hacerlo en una gran ciudad. En el grupo de los integrados, NSE alto y medio, en lugar de dos, la segmentación conforma tres grupos, siempre en 
razón directa del tamaño de la urbe o localidad que se habita. Para los grupos de menores ingresos (rezagados) habitar una gran ciudad, le brindaria mayores posibilidades a recursos tecnológicos generalmente sustentados en su capacidad de endeudamiento y acceso a la diversidad de opciones de crédito; disponibilidad y acceso que sus pares que viven en pueblo o ciudad pequeña no tienen o se encuentra limitada.

A su vez, en el NSE alto y medio $(\mu=0,7 ; \sigma=0,16)$ amparados en su mayor capacidad adquisitiva, acceso al crédito y/o influencia de la publicidad, les posibilita diferenciarse con mayor intensidad con base al tamaño de la zona que se habita. En este segmento, el habitar una gran ciudad facilita la penetración tecnológica respecto de vivir en zonas menos urbanizadas y/o pobladas $(\mu=0,74 ; \sigma=0,14)$. Así mismo, quienes habitan en una ciudad pequeña $(\mu=0,63 ; \sigma=0,15)$ y que pertenecen al mismo segmento se diferencian respecto de aquello que lo hacen en un pueblo o campo $(\mu=0,55 ; \sigma=0,11)$, pero estos últimos aún teniendo menos equipamiento que sus pares que habitan en espacios más densificados, logran diferenciarse de aquellos que pertenecen a un segmento de menor poder adquisitivo.

La diferencial distribución de bienes y dispositivos tecnológicos por nivel socioeconómico y lugar de residencia tiende a ser una constante observada. Así por ejemplo, en lo que respecta a la zona rural la penetración desigual es significativa respecto de otros espacios territoriales y comprende casi todos los bienes, no obstante la importancia de esta variable se ve atenuada con base al segmento socioeconómico de pertenencia.

En un tercer y último nivel aparecen los años de escolaridad, aunque su significancia tiende a ser relevante solo en algunos de los subconjuntos generados. En concreto para las mujeres de nivel socioeconómico bajo y que vivan en un pueblo, ciudad pequeña, o campo, se observan segmentaciones diferenciadas según se tenga o no siete años de escolaridad, es decir, próximo a la enseñanza básica completa (Gr. $<7$ años de escolaridad [ $\mu=0,34 ; \sigma=0,14] ;$ Gr. $>$ años de escolaridad [ $\mu=0$ 44; $\sigma=0,15 \rrbracket$.

Igual situación acontece en el grupo socioeconómico alto y medio que habita la gran ciudad pero con un mayor nivel de diferenciación, constituyéndose a partir de allí tres grupos según estén próximos a terminar la enseñanza media (11 años de escolaridad), tengan formación técnica superior incompleta (entre 12 y 14 años de escolaridad) o por último tengan estudios de nivel superior (más de 14 años de escolaridad). En este nivel, el segmento de mayor escolaridad evidencia un alto Índice de Tecnificación del Hogar $(\mu=0,82 ; \sigma=0,12)$, seguido del de escolaridad intermedia 
$(\mu=0,74 ; \sigma=0,14)$, ambos grupos fuertemente diferenciados respecto del segmento de menor escolaridad $(\mu=0,67 ; \sigma=0,13)$.

En efecto, el nivel de estudios se sitúa como un factor relevante en su influencia sobre el nivel de equipamiento doméstico, aunque esta importancia está influenciada también por el nivel de ingresos. Mayor capital humano acumulado implica por una parte, mayores rentas que posibilitan financiar bienestar y consumo, y por otra, un perfil sociocultural diferenciado que plantea otras necesidades y requerimientos. Educación que por lo demás tiende a ser diferencialmente valorada por el mercado del trabajo en razón del nivel de desarrollo productivo y económico alcanzado.

Los mayores niveles de educación y como corolario las mayores rentas se generan en las grandes ciudades y es aquí precisamente donde el mercado del trabajo discrimina con mayor intensidad por cada año de escolaridad alcanzada. Credenciales educativas que en sectores productivos menos especializados, predominantemente presentes en ciudades pequeñas, pueblos o campos, no son igualmente valoradas. De igual forma el habitar en grandes ciudades conecta a las personas con mayor facilidad a circuitos de consumo pues en este espacio existe una mayor disponibilidad de oferentes de bienes y servicios, así como mayor facilidad de acceso al endeudamiento mediante el crédito.

Similares hallazgos, aunque utilizando diseños y métodos de análisis diferenciados, los encontramos en Díaz de Rada, (2001), Brandle (2005), Boito, Michelazzo y Conicet (2011).

\section{CONCLUSIONES}

El aumento de los niveles de producción que incide en los costos, el mejoramiento y estabilización de los ingresos percibidos; sumado a la capacidad de endeudamiento y la instalación de nuevas pautas de bienestar y consumo han propiciado la irrupción masiva de tecnologías en el espacio doméstico. No obstante sus sesgos y brechas, diversos dispositivos tecnológicos han complementado el equipamiento doméstico en variedad y cantidad siendo expresión de confort y status. Desarrollo tecnológico que redefine los lujos y lo suntuario. Encontramos dispositivos de presencia marginal en el pasado, siendo hoy de acceso masivo e incluso universal. Tal es el caso del teléfono celular, la televisión a color y televisión por cable.

En términos generales se distinguen por un lado tecnologías ahorradoras de tiempo y por el otro, consumidoras del mismo. En efecto, dispositivos presentes en el hogar como la lavadora y el horno microondas simplifican tareas, lo que deriva en 
una mayor disponibilidad de tiempo, en especial para la mujer. Espacio de ocio o de tiempo extra disponible que posibilita su inversión en actividades remuneradas fuera del hogar por cuanto la productividad doméstica se ve mejorada con la incursión de estas tecnologías liberando de estas faenas al miembro del hogar encargado, tradicionalmente de sexo femenino. Por otra parte, y asociado a estas nuevas pautas de consumo, el alcanzar determinado nivel de confort que las tecnologías prometen, se precisa generalmente de una mayor cantidad de perceptores de ingresos. Ello conmina a la familia entendida como unidad de consumo a hacer uso de la fuerza de trabajo de reserva, generalmente constituida por mujeres, las cuales irrumpen en el mercado laboral a fin de generar también el ingreso que posibilite el consumo tecnológico.

Se constata una fuerte penetración de aparatos electrónicos consumidores de tiempo, los cuales son utilizados para la entretención, generalmente de usos y accesos privatizados y de consumos individuales. Tipología de dispositivos que por lo demás suele existir en más de una unidad por hogar, situación que profundiza esta tendencia.

Se distingue un grupo de electrodomésticos que no se encuentran totalmente integrados, escaso uso a nivel global y de presencia fuertemente segmentada. Como ejemplos de esta tendencia se encuentra la secadora, el congelador y el lavavajillas que son expresión de pautas de consumo y estilos de vida elitizados, donde el bienestar y el tiempo son elementos valorados significativamente, pero solo al alcance de un segmento reducido de la población.

La penetración de algunos dispositivos se hace también a expensas de otros en un contexto de sustitución e integración tecnológica cada vez más generalizada. Hecho motivado no solo por lo económicas que resultan las nuevas tecnologías sino también por su multifuncionalidad. Un ejemplo que ilustra este fenómeno es la masiva incorporación del teléfono móvil en desmedro del cada vez menos presente teléfono de red fija.

Dentro de los principales factores asociados a la penetración tecnológica en el hogar encontramos el nivel socioeconómico, el lugar de residencia y el nivel educativo, factores cuya importancia se relativiza según segmento o perfil del usuario o consumidor. Un mayor nivel socioeconómico aumenta la capacidad de compra, de endeudamiento, inmediatiza el interés de satisfacción y posibilita el deseo de diferenciación. El lugar de residencia, expresa las posibilidades de un mercado del trabajo más diversificado y con mejores rentas, y los incorpora en forma más inmediata y cercana a los flujos de consumo. El nivel educativo, mejora las 
expectativas y profundiza la tendencia de perfil aspiracional por medio del acceso a bienes y servicios que expresan bienestar y status.

\section{REFERENCIAS}

Aristegui, I., y Silvestre, M. (2012). El ocio como valor en la sociedad actual. ARBOR, Ciencia, Pensamiento y Cultura, 283-291.

Ballent, A. (1996). La publicidad de los ámbitos de la vida privada representaciones de la modernización del hogar en la prensa. Alteridades, VI (11), 53-74.

Bell, D. (2004). Las contradicciones culturales del capitalismo. Madrid: Alianza.

Boito, M., Michelazzo, C., y Conicet, C. U. (2011). Artefactos, fantasías y entornos: Una lectura sobre las tecnologias en el espacio doméstico. Córdoba: CIECyS-UE Conicet.

Borrás, V. (2007). Las desigualdades en el consumo a través del género. Revista Española de Sociología, 139-156.

Brandle, G. (2005). Consumo y cambio social en España: Evolución en el equipamiento doméstico. Reis, 120 (1), 75-114.

Brandle, G. (2008). Bienestar doméstico y cambio social en la sociedad española: El valor de los objetos en la vida cotidiana. Madrid: Universidad Complutense de Madrid.

Brandle, G. (2010). Empleo femenino y consumo doméstico: El equipamiento del hogar como modelo de análisis. Acciones e investigaciones sociales, 109-134.

Carosio, A. (2008). El género del consumo en la sociedad de consumo. Revista de estudios de género, 130-169.

Castells, M. (2006). Comunicación movil y sociedad. Barcelona: Ariel.

Corvalán, J. (2012). El ocio y tiempo libre en la sociedad actual. Contribuciones a las Ciencias Sociales.

Díaz de Rada, V. (2001). Pautas de consumo y ahorro en los albores del siglo XXI. Madrid: CIS.

Fernández, J. (2006). La sociedad del ocio: un reto para los archivos. Códices, II (2).

Flavian, C., Martínez, E., e Polo, Y. (1997). Productos consumidores vs. productos ahorradores de tiempo. Distribución y consumo, VIII (37), 146-157.

Livingstone, S. (1992). The meaning of domestic Technologies: A personal construct analysis of familial gender relations. En S. Livingstone, Consuming Technologies. Media and information in domestic space (pp. 113-130). Londres y Nueva York: Routledge.

Robinson, J., y Godbey, G. (2008). Time for life: The surprising ways americans use their time. Pennsylvania State University Press: University Park.

Santa Marina, C. (2001). La construcción de la mujer moderna en la sociedad de consumo. CIMOP.

Senet, R. (2000). La corrupción del carácter: Las consecuencias personales del trabajo en el nuevo capitalismo. Barcelona: Anagrama.

Serrano, A. (1994). El hogar y sus objetos: Un análisis semio-sociológico. Politica y Sociedad, 225-231. 